\title{
SHIELDING INTENTIONS FROM DISTRACTION: FORMING AN INTENTION INDUCES INHIBITION OF DISTRACTING STIMULI
}

Harm Veling and Ad van Knippenberg

Radboud University Nijmegen

Previous research has shown that focal goals are shielded through inhibition of alternative goals. The present research aims to extend these findings and show that execution of experimentally induced intentions is also shielded from distraction. In two experiments participants were instructed to form an intention to react to specific stimuli (intention cues). Next, we assessed accessibility of the intention cues, distracting cues and control cues. Results show that distracting cues were inhibited compared with control cues. In addition, we obtained preliminary evidence that this inhibition facilitates execution of previously formed intentions. The present research adds to earlier research on intentions and goal shielding by showing that cognitive self-regulatory processes shield intentions from distraction.

Fending off distractions to shield execution and completion of intentions is indispensable for successful goal pursuit. Preparing a lecture, reading an article or writing a paper all depend, at least partly, on the ability to not get distracted too often. Unfortunately, however, there are usually many environmental cues that might interfere with the processing of intention-related behavior and hence hinder the proper implementation of our intentions: Receiving an e-mail at work from a friend may trigger a memory representation of a party that temporarily interferes with prepar-

\footnotetext{
Address correspondence to Harm Veling, Department of Social Psychology, Behavioural Science Institute, Radboud University Nijmegen, PO Box 9104, 6500 HE Nijmegen, The Netherlands. E-mail: h.veling@psych.ru.nl.

The Netherlands Organization for Scientific Research (NWO) supported this research, Grant 575-31-002.
} 
ing the lecture, reading the article or writing the paper. Nevertheless, in many cases we appear to be able to cope with distraction and reach our intended goals. Apparently, self-regulatory processes can overcome or shield ongoing intentions from these distractions. The present article is concerned with the question of how we execute appropriate behavior in response to suitable environmental cues without interference from distracting cues.

Goal-directed behavior is in large part determined by environmental cues (see Bargh, 1990; Bargh \& Ferguson, 2000). These cues may guide behavior either directly or may be mediated by internal representations (Bargh \& Ferguson, 2000; Gollwitzer \& Moskowitz, 1996). Indeed, a growing area of research is showing that goal-related cues can activate goals, goal-directed information processing and goal-directed behavior even without awareness of these cues (e.g., Bargh, Gollwitzer, Chai, Barndollar, \& Troetschel, 2001; Chartrand \& Bargh, 1996; Moskowitz, Gollwitzer, Wasel, \& Schaal, 1999). For instance, Bargh et al. showed that priming participants with an achievement goal increased performance on an intellectual task compared with performance in a control condition. Because environmental cues play such a major role in goal-directed behavior, regulation of accessibility of these cues seems crucial for successful goal attainment. Once a goal is chosen, it is important to shield this goal from interference of distracting stimuli. Presumably then, goal attainment is facilitated by inhibition of interfering stimuli such as alternative goals, temptations or distracting environmental cues (Kruglanski et al., 2002; Mischel, Cantor, \& Feldman, 1996).

Consistent with this reasoning, recent research on goal shielding has shown that activation of a focal goal causes inhibition of alternative goals (Shah, Friedman, \& Kruglanski, 2002). More specifically, Shah et al. showed that activation of a self-relevant goal to which participants are committed causes inhibition of alternative goals that could interfere with attainment of the self-relevant goal. Thus, at the level of goal representations it is clear that activation of a desired goal causes inhibition of interfering goals. However, the process of goal striving does not end with activation of an (abstract) focal goal. Successful goal pursuit also relies on self-regulatory skills in initiating, executing and completing goals (Gollwitzer, 1999; Mischel, Cantor, \& Feldman, 1996). 
So, after goal selection, planning of goal attainment becomes imperative (e.g., Heckhausen \& Gollwitzer, 1987; Gollwitzer, 1990, 1996). During this implementation phase of goal pursuit more concrete goals are needed that deal with fulfillment of the how, where and when of goal pursuit (e.g., Heckhausen \& Gollwitzer, 1987; Gollwitzer, 1996). We will refer to such concrete goals as intentions (see Tomasello, Carpenter, Call, Behne, \& Henrike, in press). More specifically, Kuhl (1987) defines an intention as "an activated plan to which an actor committed herself or himself" (p. 282). Thus, intentions specify behaviors required for goal completion and hence differ from goals in that they are more concrete. In addition, as compared to goals, intentions are highly variable, because most goals can be achieved in numerous ways. Just as abstract goals, however, intentions require shielding from distraction in order to complete them (Gollwitzer, 1999). How can we accomplish this shielding of intentions?

On the level of goal representations, Shah, Friedman, and Kruglanski (2002) have argued that inhibition of distracting goals is learned: After repeatedly inhibiting a distracting goal in setting of a focal goal, mere activation of the focal goal inhibits the distracting goal. Such learning of inhibitory relations is possible because, at some level of abstraction (e.g., shopping, studying, partying, sporting), at a particular point in time, there may not exist so many competing goals.

However, a similar process seems unlikely for inhibition of distracting cues in the case of shielding intentions. As described above, goal implementation through the formulation of concrete intentions can be very variable. Additionally, distractions that might interfere with an intention are also highly variable. These distractions may include other intentions, distracting memory representations, and virtually any interesting salient environmental stimulus. Consequently, it seems virtually inconceivable that one could learn an inhibitory relation between every intention and every distracting stimulus. The scope of possible relations between feasible intentions and possible distractions when implementing attainment of a focal goal seems too wide and diverse to ever be learned. Postulation of a flexible mechanism that targets all kinds of interference seems necessary to explain how intentions are shielded from distraction. 
We argue that the degree of interference of a stimulus predicts the level of inhibition this interfering stimulus is subjected to. If a stimulus does not interfere with an ongoing intention there is no need to inhibit it. However, if a stimulus does interfere with an ongoing intention, shielding from this stimulus becomes necessary for successful intention completion. A comparable process has already been revealed in research on the retrieval of information from long-term memory. In particular, research on retrieval-induced forgetting (Anderson, Bjork, \& Bjork, 1994) has shown that retrieval of a specific memory representation from long-term memory can cause forgetting (e.g., Anderson, Bjork, \& Bjork, 1994; Macrae \& MacLeod, 1999) and inhibition (Anderson \& Spellman, 1995; Veling \& van Knippenberg, 2004) of related memory representations. The explanation for this paradoxical effect is that during retrieval of a specific memory representation, other related memory representations also become activated. As these related representations may interfere with retrieval of the intended memory representation, inhibitory processes suppress the related interfering representations, thereby facilitating selection of the target memory representation (Anderson \& Spellman, 1995; a similar process has also been shown on a neurological level in the context of intentional forgetting by Anderson et al. [2004]).

It may be argued that retrieval is just a special case that recruits executive control processes to override pre-potent responses (in this case, override retrieval of interfering memories; Anderson, 2003). The inhibitory mechanisms that cause inhibition of interfering memory representations are not limited to retrieval processes but, as Anderson (2003) stated, "are essential to our ability to behave in a flexible, context-appropriate manner" (p. 442). Consistent with this reasoning we argue that intentions recruit inhibitory processes to deal with interfering (and thus distracting) stimuli. Furthermore, we think that this inhibition of interference can occur spontaneously, that is, once an intention is active interference will be inhibited without an instruction to do so.

To test this idea we asked participants to form an intention and subsequently we measured the accessibility of interfering and control cues. In the present research we manipulated the degree of interference by varying strength of semantic associations be- 
tween intention relevant cues with other environmental cues. The idea is that stronger associates interfere more and therefore require inhibition (see Anderson \& Spellman, 1995). We presented participants with exemplars from two semantic categories (fruits and animals). Next, we asked participants to form an intention to respond to a selection of exemplars of one of the categories (e.g., peach, melon and strawberry). After that, we measured the accessibility of cues from the same semantic category as the intentional cues (e.g., grape, plum and orange - the distracting cues) and cues from an unrelated semantic category (e.g., elephant, rabbit and lion; i.e., the control cues) during an intention execution task. Our hypothesis is that cues that are semantically related to the intentional cues are more likely to interfere with the content of the intention and hence require more inhibition compared with cues from an unrelated semantic category. We tested this hypothesis in two experiments.

\section{THE PRESENT PARADIGM}

In both experiments participants first memorized exemplars of two categories (e.g., fruit-peach, fruit-grape, animal-lion, animal-elephant). This phase was included to activate all stimuli in memory. In the second phase participants received a modified lexical decision task. Participants were instructed to do a lexical decision task and in addition to form an intention to press the space bar after each lexical decision concerning a subset of exemplars from one of the categories. Pressing a designated key in response to a specific cue has been used previously to operationalize intentions (e.g., Marsh, Hicks, \& Cook, 2005). This manipulation generated three types of cues: exemplars that require a space bar response after the lexical decision (intention cues, for example peach), exemplars from the same semantic category as the intention cues that do not require a space bar response (distracting cues, for example, grape), and exemplars from an unrelated category that do not require a space bar response (control cues, for example, lion). Note that the instruction in the first phase to memorize all the exemplars enhanced the probability that the distracting cues would require inhibition during this lexical decision task. By means of lexical decision latencies to the different 
cues we assessed the accessibility of all memorized exemplars. We hypothesize slower lexical decision latencies to distracting cues compared with lexical decision latencies to control cues. Moreover, we explored whether this inhibition facilitates the execution of the intentions (space bar presses).

\section{EXPERIMENT 1}

\section{METHOD}

Participants. Seventy-three undergraduate students from the Radboud University Nijmegen participated in Experiment 1 in exchange for $€ 2$ (approximately \$2.60).

Materials. Two categories (fruit and animals) each comprising six exemplars were constructed for Experiment 1. These category-exemplar pairs were selected from a study on category norms in which Dutch category-exemplar pairs were generated (Storms, 2001). ${ }^{1}$ The exemplars of each category were matched for category typicality and word length. To ensure that every exemplar would function as an intention cue, distracting cue or control cue, four sets were constructed in which the function of exemplars varied between participants. Three exemplars of one category served as intention cues within each set, leaving the other three exemplars from this selected category as distracting cues. The exemplars from the other category served as control cues.

Two types of fillers were used in the lexical decision task. First, five words were included that did not belong to any of the categories. These words were presented within the first ten trials in order to familiarize the participants with the lexical decision task. In addition we used 53 non-words as fillers to ensure that the probability of a word response equaled the probability of a non-word response. These were 53 pronounceable letter strings, which were not part of the Dutch vocabulary. All fillers were matched to the length of the experimental cues.

Procedure. In the present experiments participants read self-paced through the instructions. The first instructions screen informed the participants that the experiment consisted of three

1. All stimuli can be obtained from Harm Veling. 
phases. In the first phase, participants were asked to study word pairs from two categories: fruits and animals. Participants were informed that they would be asked to retrieve those word pairs in the final phase of the experiment. Category exemplar pairs were then presented one by one for $5 \mathrm{sec}$ with a $1 \mathrm{sec}$ interval between presentations. The categories were presented in capitals and were separated by a dash from the exemplars, which were presented in lower case letters (e.g., FRUIT - mango). The category-exemplar pairs of each category were presented as a block. The order of these blocks and the presentation of the exemplars within each block were randomized.

The second phase of the experiment was presented as a study on memorizing intentions. First, participants received instructions for the lexical decision task. They were instructed to indicate as fast and as accurately as possible whether letter strings, presented in the middle of the computer screen, were words. Participants were instructed to press the left button (the F) when a non-word was presented and the right button (the K) when a word was presented, on a QWERTY keyboard. An asterisk that was presented for 1.5 to $2 \mathrm{sec}$ indicated the spot where letter strings would appear. Following a participant's response a backward mask (XXXXXX) replaced the letter string. This mask remained on the screen for $1.5 \mathrm{sec}$.

Subsequently, participants read that they were required to press the space bar after lexical decisions concerning three specific words from one of the categories presented in the first phase of the experiment. This space bar response could be executed during the presentation of the backward mask. The instruction was: After your lexical decision concerning peach, melon and mango we need you to press the space bar (the presented intention cues depended on the stimulus set participants received). Moreover, to ensure fast and accurate responses, we promised to reward the fastest, most accurate participant with a book token of 10. After this instruction participants read a summary of all instructions, which emphasized that the occasional space bar response should occur after the lexical decision. Furthermore, participants read that they should use a thumb of their hand of choice to press the space bar. They were asked to keep their index fingers on the $\mathrm{F}$ and $\mathrm{K}$ buttons and their thumbs on the space bar during the reaction time task. After 
reading these instructions they could press a button to start the reaction time task.

The reaction time task consisted of 106 trials. All intention cues and all distracting cues were presented four times each (24 trials in total) and the control cues were presented twice (24 trials in total). Thus, the reaction time task consisted of 106 trials (12 intention cues, 12 distracting cues, 24 control cues, 5 filler words and 53 filler non-words). Two orders of trial presentation were constructed (control cue, non-word, intention cue, etc). Within these orders the stimuli were randomly selected and one of these orders was randomly selected between participants.

The reaction time task was followed by a free recall task in which participants were cued with the category labels and were asked to write down as many of the exemplars from the first phase of the experiment as possible. Note that this free recall task was merely inserted as a follow up to the memorize instructions given earlier in the experiment.

\section{RESULTS AND DISCUSSION}

Two participants were excluded from the following analyses because they pressed the space bar on every trial, leaving 71 participants for analyses. In Experiments 1 and 2 we used several data transformations to deal with outliers (i.e., a cut-off criterion of $2 \mathrm{~s}$, a cut-off-criterion of $3 \mathrm{~s}$ or removal of response latencies beyond 3 SDs from the mean). The following analyses are reliable in all these transformations. The reported analyses and means are based on the $3 \mathrm{~s}$ cut-off criterion (retaining $99.7 \%$ of the data distribution in both experiments). Furthermore, we excluded trials in which lexical decisions were incorrect $(2.3 \%)$ and trials in which participants made an erroneous space bar response $(1.8 \%)$.

To test whether intentions caused inhibition of distracting cues we performed a single factor (cue type: intention, distracting, control) within-subjects analysis of variance (ANOVA) on the lexical decision latencies. As expected, the main effect of cue type was reliable, $F(2,140)=4.41, p<.05$. Simple effect tests revealed that this effect was caused by increased reaction times to the distracting cues $(M=698 \mathrm{~ms}, S D=178)$ compared with both the intention 
cues $(M=672 \mathrm{~ms}, S D=165)$ and control cues $(M=668 \mathrm{~ms}, S D=$ 113), respective comparisons $F(1,70)=10.53, p<.05, d=.15$, and $F(1,70)=5.42, p<.05, d=.20$. There was no reliable difference between the intention and control cues, $F(1,70)<1$, ns. The increased lexical decision latencies to the distracting cues demonstrate that cues that interfere with an intention are indeed inhibited. As such, these results are consistent with the idea that distracting stimuli are inhibited to shield the implementation of intentions.

Next, we assessed whether this inhibition of distracting cues was helpful in executing the space bar response after lexical decisions to intention cues. To examine this functionality of the observed inhibition of distraction, we computed a correlation between the strength of inhibition (calculated as the difference between lexical decision latencies to distracting cues and lexical decision latencies to control cues) and goal directed behavior (latencies of space bar presses), partialling out the mean lexical decision latencies on all trials (to control for individual differences in reaction times). This analysis yielded a reliable negative partial correlation between these two variables, $\operatorname{pr}(68)=-.28, p<$ .05. This correlation indicates that stronger inhibition of distraction is related to faster space bar pressing. Thus, it appears that intentional behavior (space bar presses) is facilitated if distracting cues are inhibited more strongly. This partial correlation corroborates a functional account of the inhibition of distracting cues, by showing that protecting the content of intentions, through inhibition of distraction, facilitates execution of these intentions.

At first sight it may seem remarkable that accessibility of intention cues was not facilitated compared with accessibility of control cues. Indeed, studies in the domain of implementation intentions, for instance, have reported increased accessibility of intention relevant cues (e.g., Aarts, Dijksterhuis, \& Midden, 1999; for a review see Gollwitzer \& Sheeran, 2004). However, the pattern of the present results is consistent with prior research (Veling \& van Knippenberg, 2004, Experiment 2) that also reported inhibition of distracting cues, but no facilitation in lexical decision latencies of cues with a special status (in that case repeatedly retrieved cues). Presumably, explicitly memorizing cues produces asymptotic levels of accessibility of both intention and control cues which leads to ceiling effects concerning these cues in the 
subsequent lexical decision task. As a result, the lexical decision task only reveals inhibition of distracting cues. Similarly, forming an intention may not necessarily enhance accessibility of intention cues compared with (also presented) control cues. Consequently, the absence of facilitation of intention cues in Experiment 1 should not be interpreted as intention cues having no special status (Gollwitzer \& Sheeran, 2004; see also Goschke \& Kuhl, 1993), but should be attributed to the nature of the dependent measure in combination with memorized words.

\section{EXPERIMENT 2}

In Experiment 2 we aimed to replicate the findings of Experiment 1. Moreover, because in everyday life distractions are unexpected and maybe infrequent, we examined whether the inhibition of distracting cues would still hold in a more complex situation. To examine this, participants in Experiment 2 received five intention cues that were only presented once in a total of 92 trials. So whereas the probability of encountering an intention cue in Experiment 1 was $11.3 \%$, this probability was reduced to $5.4 \%$ in Experiment 2. In addition, a specific intention cue was presented only once in Experiment 2 compared with four times in Experiment 1 . We expected the same pattern of results as we have observed in Experiment 1.

\section{METHOD}

Participants. Fifty-five undergraduate students from the Radboud University Nijmegen participated in Experiment 2 in exchange for $€ 2$ (approximately \$2.60).

Materials. Two categories (fruit and animals) each comprising ten exemplars were constructed for Experiment 2. These exemplars were again selected from the sample from Storms (2001) and the categories were matched following the same criteria as used in Experiment 1. To ensure that every exemplar would function as an intention, distracting or control cue, four sets were constructed that varied the function of exemplars between participants. Five exemplars of one category served as intention cues within each set, leaving the other five exemplars from this selected category as 
distracting cues. The exemplars from the other category served as control cues.

Two types of fillers were used in the lexical decision task. First, there were 27 words that did not belong to any of the categories. These were included to separate the experimental exemplars by at least three trials. Second, we used 45 non-words. These were 45 pronounceable letter strings, which were not part of the Dutch vocabulary. All fillers were matched to the length of the experimental exemplar words.

Procedure. The procedure of Experiment 2 was virtually identical to that of Experiment 1 . The main difference was that participants memorized 20 category exemplar pairs and that they were instructed to form an intention to respond to five intention cues in the second phase of the experiment (half of the exemplars from one of the categories). The instruction read: After lexical decisions concerning five specific words we need you to press the space bar. These words will be presented when you press a button. These five intention cues were simultaneously presented on the screen for 30 seconds. In addition, the lexical decision task was modified such that each experimental cue was presented only once. Thus, the reaction time task consisted of 92 trials ( 5 intention cues, 5 distracting cues, 10 control cues, 27 filler words and 45 filler non-words).

\section{RESULTS AND DISCUSSION}

Two participants were excluded from the analysis because they pressed the space bar on every trial, leaving 53 participants for analysis. To deal with outliers we used the same data transformations as in Experiment 1. In Experiment 2, 2.6\% of the lexical decisions were incorrect and $2.8 \%$ of the space bar responses were incorrect. These responses were again omitted from the following analyses.

We performed a single factor (cue type: intention, distracting, control) within-subjects analysis of variance (ANOVA) on the lexical decision latencies to assess whether intentions caused inhibition of distracting cues. As expected, the main effect of cue type was again reliable $F(2,104)=4.28, p<.05$. Simple effect tests revealed that this effect was due to increased reaction times to the 
distracting cues $(M=890 \mathrm{~ms}, S D=324)$ compared with both the intention cues $(M=839 \mathrm{~ms}, S D=248)$ and control cues $(M=811$ $\mathrm{ms}, S D=190)$, respective comparisons $F(1,52)=4.40, p<.05, d=$ .18 and $F(1,52)=5.66, p<.05, d=.30$. There was no reliable difference between the intention and control cues, $F(1,52)=1.41$, ns. These results nicely replicate the results of Experiment 1 and indicate that cues that are related to intention cues are inhibited.

To assess the functionality of the reported inhibition effect we computed the same correlation as in Experiment 1. This partial correlation between the strength of inhibition and reaction times on space bar presses while controlling for individual differences in overall reaction time speed was marginally reliable, $\operatorname{pr}(50)=$ $-.27, p=.055$. This correlation is of the same magnitude as the correlation in Experiment 1 and is an indication that successful goal directed behavior (space bar presses) is directly related to inhibition of distracting cues.

\section{GENERAL DISCUSSION}

We conducted two experiments to examine the shielding of intentions from distracting stimuli. We expected that forming an intention would cause inhibition of semantically related and thus distracting cues compared with unrelated control cues. Both experiments confirmed that instructing participants to react to specific cues (intention cues) inhibits access to cues that might interfere with this intention (distracting cues) compared with control cues. This pattern of results is consistent with the idea that cognitive self-regulatory processes spontaneously shield intentions from distraction.

We would like to emphasize that the main aim of the reported experiments was to show inhibition of distracting cues. Nonetheless, we also found some preliminary support for a functional account of this inhibition effect in Experiments 1 and 2: As inhibition of distracting cues was stronger, participants were faster to execute their intentions. Although the correlation is

moderate, it nevertheless nicely illustrates the functionality of the inhibition of distracting cues.

The effect sizes of the inhibition of distracting cues as indicated by Cohen's d are small. However, it should be noted that these in- 
hibition effects resulted from relatively subtle manipulations. In both experiments, participants were simply instructed to press the space bar after encountering intention cues during a lexical decision task. Even though the intention cues were displayed only once on the screen, lexical decisions to distracting cues were slower compared to control cues. Accordingly, considering such minimal inductions of intention, it is not surprising that the effects sizes obtained are rather small.

The present results are consistent with, but also different from, research on goal shielding by Shah et al. (2002). Just as activating a focal goal inhibits alternative goals, forming an intention induces inhibition of distracting stimuli. However, the explanation for inhibition of alternative goals offered by Shah et al., that is, overlearned inhibition of the alternative goal in pursuit of a focal goal, cannot explain the present results. Participants inhibit a word like grape in pursuit of pressing the space bar to orange. Because participants have probably never been in pursuit of this specific intention, inhibition of distraction is unlikely to be learned. Therefore, we propose a basic mechanism that can explain inhibition of distraction concerning new intentions: Any content that interferes with the content of an intention will receive inhibition. Consistent with this idea we found that stimuli merely semantically related to the content of an intention are inhibited compared with (non-distracting) semantically unrelated stimuli. Note, however, that the processes underlying shielding goals versus shielding intentions are not mutually exclusive, but complementary. In goal selection learned inhibitory relations inhibit alternative goals, whereas in goal execution intentions are shielded through inhibition of any interference. Thus, the present results give new insight into the path from goal setting to goal execution.

The reasoning from the previous paragraph provides an interesting avenue for future research. One implication might be that if one specific distracting stimulus keeps interfering with one specific intention, an inhibitory relation develops between this intention and the distracting stimulus. Interestingly, such a process could explain how overlearned inhibitory relations between competing goals develop, i.e., inhibitory relations originate from re- 
peated interference (Shah et al., 2002; see also Fishbach, Friedman, \& Kruglanski, 2003).

Because the paradigm used in the present research is adapted from research studying long-term memory processes, more specifically part-list cuing (e.g., Basden \& Basden, 1995) and retrieval-induced forgetting (Anderson, Bjork, \& Bjork, 1994; Veling \& van Knippenberg, 2004), it is important to note that such previously reported long-term memory processes cannot account for the present data. ${ }^{2}$ Part-list cuing refers to the phenomenon that presenting participants with part of a previously studied list impairs recall of the whole list compared with a condition in which no part of the list is presented. Part-list cuing effects are likely caused by a disruption of retrieval processes when part of the list is available during retrieval of the whole list (Basden \& Basden, 1995). The key issue is that part-list cuing is not obtained when the presentation of part of the list (in our case presentation of intention cues) and measurement of memory for the whole list (in our case in the lexical decision task) are separated (for a discussion of these whole-part-whole experiments see Basden and Basden, 1995). Moreover, because our main dependent variable (lexical decision) taps accessibility rather than retrieval processes, part-list cuing is further eliminated as an underlying process. Thus, another process is required to account for the inhibition of distracting cues.

In a related vein, research on retrieval-induced forgetting has repeatedly shown that when part of a previously studied list and the whole studied list are presented in separated phases of an experiment, mere presentation of part of the list is not sufficient to obtain any inhibition when memory for the whole list is tested (Anderson, Bjork, \& Bjork, 2000; Bäuml, 2002). Based on these arguments we argue that our results do not merely reflect an old long-term memory phenomenon, but are new because they show that inhibition of part of the list (i.e., the inhibition of distracting cues) can be found when it interferes with keeping an intention in mind.

2. We would like to thank Jim Uleman and an anonymous reviewer for drawing our attention to this issue. 
The current data have interesting implications for theorizing on the status of intentions in memory. Previous research on this subject suggests that intentions are in a constant state of heightened activation (the intention superiority effect; Goschke \& Kuhl, 1993; Marsh, Hicks, \& Bink, 1998). However, this previous research is based on a comparison between two kinds of stimuli: intention stimuli and other to be remembered stimuli. On the basis of our research it seems arguable that the to be remembered stimuli are inhibited to prevent them from interfering with the intention stimuli. This could mean that intentions are either in a heightened state of activation, or potentially interfering stimuli are in a lowered state of activation, or both. However, because the stimuli and research procedures from the present experiments differ substantially from these earlier experiments, it is difficult to draw conclusions based on this comparison. Nevertheless, future research on the intention superiority effect (Goschke \& Kuhl, 1993) may include a control condition to reveal facilitory and inhibitory effects independently.

The reported experiments aimed to show that intentions spontaneously shield the content of concrete intentions from distraction by inhibiting interfering content. We obtained repeated evidence that intentions can indeed induce inhibition of cues that might interfere with intention execution. Furthermore, this inhibition seems functional because it is positively related to task performance.

\section{REFERENCES}

Aarts, H., Dijksterhuis, A., \& Midden, C. (1999). To plan or not to plan: Goal achievement or interrupting the performance of mundane behaviors. European Journal of Social Psychology, 29, 971-979.

Anderson, M. C. (2003). Rethinking interference theory: Executive control and the mechanisms of forgetting. Journal of Memory and Language, 49, 415-445.

Anderson, M. C., Bjork, R. A., \& Bjork, E. L. (1994). Remembering can cause forgetting: Retrieval dynamics in long-term memory. Journal of Experimental Psychology: Learning, Memory, and Cognition, 20, 1063-1087.

Anderson, M. C., Bjork, E. L., \& Bjork, R. A. (2000). Retrieval-induced forgetting: Evidence for a recall-specific mechanism. Psychonomic Bulletin and Review, 7, 522-530.

Anderson, M. C., Ochsner, K., Kuhl, B., Cooper, J., Robertson, E., Gabrieli, S.W., 
Glover, G., \& Gabrieli, J. D. E. (2004). Neural systems underlying the suppression of unwanted memories. Science, 303, 232-235.

Anderson, M. C., \& Spellman, B. A. (1995). On the status of inhibitory mechanisms in cognition: Retrieval as a model case. Psychological Review, 102, 68-100.

Bargh, J. A. (1990). Auto-motives: Preconscious determinants of social interaction. In E. T. Higgins \& R. M. Sorrentino (Eds.), Handbook of motivation and cognition (Vol. 2, pp. 93-130). New York: Guilford.

Bargh, J. A., \& Ferguson, M. L. (2000). Beyond behaviorism: On the automaticity of higher mental processes. Psychological Bulletin, 126, 925-945.

Bargh, J. A., Gollwitzer, P. M., Chai, A. L., Barndollar, K., \& Troetschel, R. (2001). Automated will: Nonconscious activation and pursuit of behavioral goals. Journal of Personality and Social Psychology, 81, 1014-1027.

Basden, D. R., \& Basden, B. H. (1995). Some tests of the strategy disruption interpretation of part-list cuing inhibition. Journal of Experimental Psychology: Learning, Memory, and Cognition, 21, 1656-1669.

Bäuml, K.-H. (2002). Semantic generation can cause episodic forgetting. Psychological Science, 13, 357-361.

Chartrand, T. L., \& Bargh, J. A. (1996). Automatic activation of social information processing goals: Nonconscious priming reproduces effects of explicit conscious instructions. Journal of Personality and Social Psychology, 71, 464-478.

Fishbach, A., Friedman, R. S., \& Kruglanski, A. W. (2003). Leading us not into temptation: Momentary allurements elicit overriding goal activation. Journal of Personality \& Social Psychology, 84, 296-309.

Gollwitzer, P. M. (1990). Action phases and mind-sets. In E.T. Higgins \& R.M. Sorrentino (Eds.), The handbook of motivation and cognition: Foundations of social behavior (Vol. 2, pp. 53-92). New York: Guilford Press.

Gollwitzer, P. M. (1996). The volitional benefits of planning. In P.M. Gollwitzer \& J.A. Bargh (Eds.), The Psychology of Action (pp. 287-312). New York: Guilford Press.

Gollwitzer, P. M. (1999). Implementation intentions: Strong effects of simple plans. American Psychologist, 54, 493-503.

Gollwitzer, P. M., \& Moskowitz, G. B.(1996). Goal effects on action and cognition. In E.T. Higgins \& A. W. Kruglanski (Eds.), Social psychology: Handbook of basic principles (pp. 361-399). New York: Guilford Press.

Gollwitzer, P. M., \& Sheeran, P. (2004). Implementation intentions and goal achievement: A meta-analysis of effects and processes. Manuscript submitted for publication.

Goschke, T., \& Kuhl, J. (1993). Representation of intentions: Persisting activation in memory. Journal of Experimental Psychology: Learning, Memory, and Cognition, 19, 1211-1226.

Heckhausen, H., \& Gollwitzer, P. M. (1987). Thought contents and cognitive functioning in motivational vs. volitional states of mind. Motivation $\mathcal{E}$ Emotion, 11, 101-120. 
Kruglanski, A. W., Shah, J. Y., Fishbach, A., Friedman, R., Chun, W. Y., \& Sleeth-Keppler, D. (2002). A theory of goal-systems. In M. P. Zanna (Ed.), Advances in Experimental Social Psychology (Vol. 34, pp. 331-376). New York: Academic Press.

Kuhl, J. (1987). Action control: The maintenance of motivational states. In F. Halish \& J. Kuhl (Eds.), Motivation, intention and volition (pp. 279-291). Berlin: Springer-Verlag.

Macrae, C. N., \& Macleod, M. D. (1999). On recollections lost: When practice makes imperfect. Journal of Personality and Social Psychology, 77, 463-473.

Marsh, R. L., Hicks, J. L., \& Bink, M. L. (1998). Activation of completed, uncompleted, and partially completed intentions. Journal of Experimental Psychology: Learning, Memory, and Cognition, 24, 350-361.

Marsh, R. L., Hicks, J. L., \& Cook, G. I. (2005). On the relationship between effort toward an ongoing task and cue detection in event-based prospective memory. Journal of Experimental Psychology: Learning, Memory, and Cognition, 31, 68-75.

Mischel, W., Cantor, N., \& Feldman, S. (1996). Principles of self-regulation: The nature of willpower and self-control. In E. T. Higgins and A. W. Kruglanski (Eds.), Social psychology: Handbook of basic principles (pp. 329-360).

Moskowitz, G. B., Gollwitzer, P. M., Wasel, W., \& Schaal, B. (1999). Preconscious control of stereotype activation through chronic egalitarian goals. Journal of Personality and Social Psychology, 77, 167-184.

Shah, J. Y., Friedman, R., \& Kruglanski, A. W. (2002). Forgetting all else: On the antecedents and consequences of goal shielding. Journal of Personality and Social Psychology, 83, $1261-1280$.

Storms, G. (2001). Flemish category norms for exemplars of 39 categories: A replication of the Battig and Montague (1969) category norms. Psychologica Belgica, 41, 145-168.

Tomasello, M., Carpenter, M., Call, J., Behne, T. \& Henrike, M. (in press). Understanding and sharing intentions: The origins of cultural cognition. Behavioral and Brain Sciences.

Veling, H., \& van Knippenberg, A. (2004). Remembering can cause inhibition: Retrieval-induced inhibition as cue independent process. Journal of Experimental Psychology: Learning, Memory, and Cognition, 30, 315-318. 